\title{
Regional inequality, spatial spillover effects, and the factors influencing city-level energy-related carbon emissions in China
}

\author{
SU Wensong ${ }^{1,2,3}$, "LIU Yanyan ${ }^{4}$, WANG Shaojian" ${ }^{5}$, ZHAO Yabo ${ }^{6}$, \\ SU Yongxian ${ }^{7}$, LI Shijie
}

1. Institute of Geographic Sciences and Natural Resources Research, CAS, Beijing 100101, China;

2. University of Chinese Academy of Sciences, Beijing 100049, China;

3. Zhongguancun Development Group Co., Ltd., Beijing 100080, China;

4. School of Geography and Tourism, Guangdong University of Finance and Economics, Guangzhou 510320, China;

5. Guangdong Provincial Key Laboratory of Urbanization and Geo-simulation, School of Geography and Planning, Sun Yat-sen University, Guangzhou 510275, China;

6. School of Architecture and Urban Planning, Guangdong University of Technology, Guangzhou 510090, China;

7. Guangzhou Institute of Geography, Guangzhou 510070, China

\begin{abstract}
Data show that carbon emissions are increasing due to human energy consumption associated with economic development. As a result, a great deal of attention has been focused on efforts to reduce this growth in carbon emissions as well as to formulate policies to address and mitigate climate change. Although the majority of previous studies have explored the driving forces underlying Chinese carbon emissions, few have been carried out at the city-level because of the limited availability of relevant energy consumption statistics. Here, we utilize spatial autocorrelation, Markov-chain transitional matrices, a dynamic panel model, and system generalized distance estimation (Sys-GMM) to empirically evaluate the key determinants of carbon emissions at the city-level based on Chinese remote sensing data collected between 1992 and 2013. We also use these data to discuss observed spatial spillover effects taking into account spatiotemporal lag and a range of different geographical and economic weighting matrices. The results of this study suggest that regional discrepancies in city-level carbon emissions have decreased over time, which are consistent with a marked spatial spillover effect, and a 'club' agglomeration of high-emissions. The evolution of these patterns also shows obvious path dependence, while the results of panel data analysis reveal the presence of a significant U-shaped relationship between carbon emissions and per capita GDP. Data also show that per capita carbon emissions have increased in concert with economic growth in most cities, and that a high-proportion of secondary industry and extensive investment growth have also exerted significant positive effects on city-level carbon emissions across China. In contrast, rapid population agglomeration, improvements in technology, increasing trade openness, and the accessibility and density of roads have all played a role in
\end{abstract}

Received: 2017-05-01 Accepted: 2017-09-01

Foundation: National Natural Science Foundation of China, No.41601151; Guangdong Natural Science Foundation, No.2016A030310149

Author: Su Wensong (1982-), PhD, specialized in urban geography. E-mail: suws.13b@igsnrr.ac.cn

"Corresponding author: Liu Yanyan (1985-), PhD, E-mail: liuyyan@mail2.sysu.edu.cn 
inhibiting carbon emissions. Thus, in order to reduce emissions, the Chinese government should legislate to inhibit the effects of factors that promote the release of carbon while at the same time acting to encourage those that mitigate this process. On the basis of the analysis presented in this study, we argue that optimizing industrial structures, streamlining extensive investment, increasing the level of technology, and improving road accessibility are all effective approaches to increase energy savings and reduce carbon emissions across China.

Keywords: carbon emissions; spatial spillover effects; dynamic spatial panel data model; Chinese carbon emission reduction policies; environmental Kuznets curve

\section{Introduction}

Changes in global climate are most notably manifest as global warming, and are the result of the large-scale consumption of fossil fuels. This effect has become the most critical international environmental issue; since the signing of the 'Kyoto Protocol', a low carbon mode of economic development has gradually been accepted as important by most countries, while participating states at the 2009 Copenhagen World Climate Conference reached a basic consensus on emission reductions. China is the first developing country to propose energy saving and emission reduction aims; the Chinese government has committed to reduce the intensity of national carbon emissions to between $55 \%$ and $60 \%$ of the 2005 level by 2020 (Wang et $a l ., 2017)$. In November 2014, the government also announced that China intends to reach a carbon emission peak around 2030 and that efforts will be made to realize this maximum level as soon as possible (The China-U.S. Joint Statement on Climate Change; Liu et al., 2016; Wang et al., 2016c, 2016d). In addition, numerous local governments within China have also developed their own low-carbon action plans, highlighting national determination in this area.

China has developed into one of the largest carbon emitting countries in the world because of the huge national level of energy consumption and the combined effects of an extensive model of development, an economic structure that emphasizes secondary industries, poor performance at reducing emissions, and inefficient environmental government (Cheng et al., 2013). A combination of energy savings and emission reductions are key to slowing global warming, and are also critical pathways for transforming the mode of economic development and upgrading industrial structures. However, the socioeconomic factors that are also implicated in carbon emissions are numerous (Zhang et al., 2013); thus, in the context of reducing emissions, it is helpful to both identify and quantify the factors that influence carbon emissions as well as their mechanisms in order to effectively formulate practical measures for mitigation (Deng et al., 2014). Research in this area is problematic, however, because the Chinese national department of statistics only discloses consumption balance tables for provinces and a few developed cities because of the national top-down energy system. Current research on Chinese carbon emissions is therefore restricted at both the national and regional level, and systematic studies on the mechanisms that influence emissions and strategies to reduce city-level $\mathrm{CO}_{2}$ production have rarely been undertaken ( $\mathrm{Su}$ et al., 2013). Previous research has nevertheless demonstrated that between $60 \%$ and $80 \%$ of global energy is consumed by urban areas, and that more than $70 \%$ of greenhouse gas emissions around the world are derived from these areas. Urban areas have therefore become key targets for energy-saving and emission-reduction initiatives (GEA, 2012; IEA, 2012), and it 
is of paramount theoretical and practical significance to guide the development of lowcarbon economies to perform research on the mechanisms that influence carbon emission reduction strategies by considering cities as basic analytical units.

Domestic and international scholars have carried out a great deal of recent research on carbon emissions and their development at different scales by applying four distinct methods. The first of these research themes has been focused on distinguishing the factors that influence carbon emissions via their structural decomposition analysis (SDA), an approach that is based on the analysis of input-output tables and models (Zhang, 2009; Wang, 2014; Wang et al., 2014a). For example, Wang et al. (2016a) performed research on the factors that influence carbon emissions in Xinjiang Province using improved IO-SDA technology and demonstrated that economy size and population are the key indexes that control carbon emission fluctuations. Similarly, in an earlier study, Feng et al. (2015) noted that economic growth in the United States between 1997 and 2007 was the most significant factor underlying increasing carbon emissions, while a change in energy structure between 2007 and 2013 was important because it led to a decrease. This kind of research is limited, however, because it is reliant on input-output tables for discontinuous intertemporal analyses; the influence of multiclass socioeconomic indicators cannot be fully investigated because the types of influencing factors included are limited. In contrast, the second research theme in this area has been founded on analysis of the extended and improved Kaya identity to understand the elements of energy consumption that make up carbon emissions via techniques such as index decomposition analysis (IDA) (He et al., 2012; Zhu et al., 2016). Thus, using LMDI for example, Wang et al. (2016b) were able to demonstrate that both population size and economic output efficiency have contributed to increasing carbon emissions from energy consumption in Xinjiang Province, while energy substitution and structural effects have contributed to reductions. Similarly, Yao et al. (2014) used IDA to show that economic and population growth are the most significant factors driving growth in the carbon emissions of G20 countries, while increases in energy efficiency and reductions in the intensity of emissions are key to curbing the ever expanding release of carbon into the atmosphere (Yao et al., 2014). The IDA method cannot be used, however, to perform residual decomposition of carbon emissions via identical transformation, while associated socioeconomic factors (e.g., transportation and trade) have seldom been taken into account (Yuan et al., 2013). The third research theme in this area is based on application of the computable general equilibrium model (CGE) which can be used to analyze combinations of economic structural optimization, levels of technology, the rate of clean energy alternatives, and differences in reduction policies. However, although the relationship between socioeconomic factors and carbon emissions can be simulated using a CGE model under general equilibrium conditions, these analyses are again based mainly on input-output tables, and it is therefore difficult to study variations in carbon emissions over time (Fan et al., 2010; Wang et al., 2015a, 2015b, 2015c). The fourth theme in this research area is based on the use of econometric models applied to analyze the different factors that influence carbon emissions (Wang et al., 2014a, 2015a) and includes metrological analyses that are applied to determine the time series of socioeconomic factors so that the dynamic effects of multiple indicators on carbon emissions can be investigated. It is noteworthy, however, that the four methods outlined above largely treat carbon emission units as homogeneous entities that are independent of one another and so ignore any spatial links and correlations. Although a small number of studies have ad- 
dressed this issue (Zheng et al., 2011; Cheng et al., 2013), this research area comprises a form of spatial econometrics which does not take into account the 'path dependence' characteristics of carbon emissions or the 'endogenous' problem of the explanatory variable. At the same time, a spatial weight approach is also the simplest kind of adjacency matrix that can be constructed, so the effects of different weight matrices on results are not taken into account (Shao et al., 2016).

Building on the background presented above, this paper addresses the emissions of city units within China by utilizing a dynamic spatial panel data model to evaluate regional differences, spatial spillover effects, and the mechanisms underlying the release of carbon into the atmosphere. The Gini coefficient and Theil index were used initially to analyze regional differences in city-level carbon emissions, and a weight matrix encompassing three kinds of distance-space (i.e., geography, economy, and geography-economy) was constructed. The spatial spillover effects of city-level carbon emissions across China were then analyzed using spatial autocorrelation and Markov-chain methods, and a comprehensive factorial analysis was also carried out using a system generalized method of moments (SGMM) approach in order to take spatiotemporal and lags, as well as spatiotemporal hysteresis effects into account. Regional differences and spatial spillover effects in city-level carbon emissions across China are also summarized in this paper and the key factors that influence carbon emissions are highlighted in order to provide a scientific basis for the development of Chinese energy saving and emission reduction policies.

\section{Data and methods}

\subsection{The spatial agglomeration characteristics of carbon emissions based on global and local autocorrelation analysis}

The spatial spillover effects of city-level carbon emissions were tested in this study via ESDA spatial autocorrelation analysis. Global spatial autocorrelation is normally performed using Moran's I coefficient, as follows (Wang et al., 2016c):

$$
I=\left[m \sum_{i=1}^{m} \sum_{j=1}^{m} \omega_{i j}\left(x_{i}-\bar{x}\right)\left(x_{j}-\bar{x}\right)\right] /\left[\sum_{i=1}^{m} \sum_{j=1}^{m} \omega_{i j} \sum_{i=1}^{m}\left(x_{i}-\bar{x}\right)^{2}\right]
$$

where $m$ denotes the number of cities in the whole country, while $\omega_{i j}$ is the spatial weight matrix, and $\bar{x}$ is the national average city-level carbon emissions. Building on this, and in order to perform a more comprehensive evaluation of the spatial correlation characteristics of city-level carbon emissions, a further two spatial weight matrices were established based on previous work. The first of these is the geospatial weight matrix $\left(W_{1}\right)$ in which weight $\left(\omega_{i j}\right)$ is represented by the reciprocal of distance between cities. Thus, taking into account the spatial associations between the economies of different cities, and to enhance the comparability of results, an economic distance weight matrix $\left(W_{2}\right)$ was also applied in this study; in this case, weight $\left(\omega_{i j}\right)$ is the inverse of the absolute difference between the per capita income of cities $i$ and $j$. However, because deviation might also occur if spatial correlation is simply weighted geographically or economically, a nested matrix $\left(W_{3}\right)$ of these weights was also applied, as follows:

$$
W_{3}=(1-\alpha) W_{1}+\alpha W_{2}
$$


where $\alpha$ ranges between 0 and 1 and reflects the proportion of weights constructed by the economy. An $\alpha$ value of 0.5 was used in this study to reflect the fact that geospatial and economic weight are thought to be equal; however, the influence of geospatial weight is also considered in $W_{3}$ which reflects the presence of a spatial spillover effect at the economic level and therefore enables the development of a comprehensive and objective correlation model for the carbon emissions of a city.

It is noteworthy that the atypical spatial characteristics of regional spaces are ignored in global spatial autocorrelation, while the use of this approach at the local level can compensate for this deficiency. Local Moran's I index is commonly used in this context, as follows (Shao et al., 2016):

$$
I_{i}=\left[\left(x_{i}-\bar{x}\right) / S^{2}\right] \cdot \sum_{j \neq i} \omega_{i j}\left(x_{j}-\bar{x}\right) ; S^{2}=\left[\sum_{i=1}\left(x_{i}-\bar{x}\right)^{2}\right] / m
$$

An $I_{i}$ value greater than zero in this expression indicates the spatial clustering of high and low carbon emission cities forms high-high and low-low patterns, while an $I_{i}$ less than zero is indicative of spatial dislocation, with cities forming high-low and low-high patterns.

\subsection{Spatial spillover effects of carbon emissions based on Markov-chains}

Spatial Markov-chain methods can be used to analyze the spatial interactions of city-level carbon emissions (i.e., spatial spillover effects). As a Markov-chain is a discrete process within this set (Pu et al., 2005), city-level per capita carbon emissions were initially divided into $\mathrm{K}$ types so that the probability of the total number occupied by each type as well as their future transition probability could be calculated (Wang et al., 2015c). Thus, the element $m_{i j t}$ denotes the probability that city-level per capita carbon emissions will revert from type $i$ to type $j$ during a year from $t$ to $t+1$. The probability matrix relationship of two adjacent years can therefore be expressed as follows:

$$
R_{t+1}=M \times R_{t}
$$

where $M$ is the transfer matrix of $K \times K$, while $R_{t}$ is the probability matrix of type $K$ in year $t$ (Wang et al., 2015c).

A spatial Markov-chain is formed from the combination of the standard method and spatial lag (Wang et al., 2015c). This kind of chain decomposes the traditional transfer matrix into a conditional one such that the element $m_{i j t}(k)$ represents the transition probability of the carbon emission type from $i$ to $j$ in the year $t+1$, where the city-level per capita carbon emission type conforms to the spatial lag condition $i$ in year $t$.

Comparing corresponding elements between spatial and non-spatial matrices makes it possible to determine the relationship between the probability of carbon emission transfer between neighboring cities. This approach also reveals the spatial spillover effects in city-level carbon emissions when different types of agglomerations neighbor one another; thus, if the type of carbon emissions from a neighboring city do not transfer in a given case, then all the corresponding elements in the two matrices will be the same (Wang et al., 2015c). Building on previous research in this area, the spatial spillover effect analysis presented here is based mainly on per capita indicators (Pu et al., 2005; Wang et al., 2015c).

\subsection{Econometric models to explore the factors determining city-level carbon emissions}

In order to more accurately reveal the factors that influence city-level carbon emission 
spillover effects, two dynamic panel data models were improved in this research to detect spatial dependence and error characteristics. City-level carbon emissions are expressed as follows (Shao et al., 2016):

$$
\begin{gathered}
C E_{i t}=\alpha_{1} X_{i t}+\rho \sum_{j} \omega_{i j} C E_{j t}+\gamma \sum_{j} \omega_{i j} C E_{j t-1}+\mu_{i t} \\
C E_{i t}=\beta_{1} X_{i t}+\xi \mu_{t-1}+\lambda \sum_{j} \omega_{i j} \mu_{i t}+\varphi_{i t}
\end{gathered}
$$

In these expressions, $C E$ refers to the per capita carbon emissions of a city, while $i$ is the city cross-section, $t$ is the year, $X$ is the eigenvector formed with an independent variable, $\alpha_{1}$ and $\beta_{1}$ are coefficient independent variable vectors, $\varphi_{i t}=\varepsilon_{i t}$ and $\mu_{i t}, \varepsilon_{i t}$, and $\mu_{i t}$ are disturbance items which conform to normal distributions, $\rho$ is the current spatial lag coefficient, $\gamma$ is the lagged one phase lag effect coefficient, and $\lambda$ is the spatial error coefficient.

The prerequisites of equations (5) and (6) are that city-level carbon emissions will change according to the factors influencing this variable and that there will be no hysteresis effect between the independent and dependent variables. In real situations, however, evolutionary trends in socioeconomic variables, including carbon emissions, exhibit obvious path dependent features; in other words, current changes are bound to be influenced by background levels at earlier times, while some of the key factors that affect city-level carbon release (e.g., population density, industrial structure, foreign direct investment) also exhibit obvious lag and dependency characteristics which may delay any expected changes in emissions. It is therefore of great practical significance to detect time lag effects in city-level carbon emissions; equations (5) and (6) were therefore locally adjusted to determine spatial hysteresis and error effects on the dynamic panel data model, as follows:

$$
\begin{gathered}
C E_{i t}=\theta C E_{i t-1}+\alpha_{1} X_{i t}+\rho \sum_{j} \omega_{i j} C E_{j t}+\gamma \sum_{j} \omega_{i j} C E_{j t-1}+\mu_{i t} \\
C E_{i t}=\theta^{\prime} C E_{i t-1}+\beta_{1} X_{i t}+\xi \mu_{t-1}+\lambda \sum_{j} \omega_{i j} \mu_{i t}+\varphi_{i t}
\end{gathered}
$$

In these expressions, $\theta$ and $\theta^{\prime}$ are the time lag coefficients used to measure the intensity of the time lag effect. As these indicate the effect of carbon emissions from the year before on current levels, it is worth noting that a dynamic spatial panel data model can be utilized to more comprehensively investigate the lag effect of city-level carbon emissions. The hysteresis effect detected in this study includes time, spatial, and spatiotemporal lags.

\subsection{Variable selection}

Environmental Kuznets curve (EKC) theory and the IPAT model were both applied in this study as they are the most common methods used to determine the factors that influence environmental stress. The panel form of the STIRPAT, an extended form of the IPAT model, is as follows:

$$
I_{i t}=a P_{i t}^{b} A_{i t}^{c} T_{i t}^{d} e
$$

where $I, P, A$, and $T$ denote environmental pressure, population data, per capita income, and technological level, respectively, while $e$ is the error term. The logarithm of both sides of this equation means that this model can be expressed as follows (Wang et al., 2016c):

$$
\ln I_{i t}=a+b \ln P_{i t}+c \ln A_{i t}+d \ln T_{i t}+e_{i t}
$$

One advantage of the STIRPAT model is that the coefficients $a, b, c$, and $d$ can be estimated as parameters, which allows for the decomposition of variables. Thus, the term $I$ in 
this expression denotes city-level per capita carbon emissions (CE), while one factor that influences the right side of this equation is population density (POPD). This variable was used to replace city-level population scale in this equation in order to explore the impact of agglomeration size on carbon emissions, as this factor usually exerts an influence via scale effects; on the one hand, an increase in population scale is bound to result in increased direct and indirect energy consumption which increases emissions, while on the other, because of population agglomeration effects, the level of science and technology will be improved to a certain extent, thereby reducing emissions. The second factor of interest that influences this relationship is economic growth (GDPPC), usually expressed on the basis of per capita gross domestic product (GDP). In order to test the traditional EKC hypothesis, the presence, or absence, of an inverted U-shaped curve can be determined between city-level per capita carbon emissions and economic growth; one of the aims of this study is to explore the relationship between carbon emissions and economic growth depending on linear and quadratic entries in per capita GDP. The third variable that is of interest in terms of the relationship expressed in equation (10) is technical level (TC). This is evaluated here on the basis of research and development investment which would be expected to increase in concert with TC even though relevant data on this variable is generally lacking at the city scale. The fourth variable of interest is industrial structure (SIND), a critical factor because the burning of fossil fuels from secondary industries (especially heavy industries) is a key component of anthropogenic carbon emissions. The ratio of added value of a secondary industry to GDP is used in this study to reflect the impact of industrial structures on city-level carbon emissions. The fifth variable of interest considered here is road network density (ROAD); this is important because when the traffic volume is the same, a higher density road network will mean lighter traffic congestion and thus a higher efficiency of vehicle exhaust emissions. Highway mileage per unit area was used in this study to explore the impact of traffic-related factors on city-level carbon emissions. The sixth variable considered of interest in this study is fixed asset investments (FAI); this variable is important because the bulk of Chinese city-level economic growth is investment-driven and so this exerts a significant impact on industrial restructuring and improvements in technological levels. City-level FAI was used in this study to evaluate the impact of carbon emissions that derive from investments. Finally, the seventh variable considered of interest in this study is 'opening up to the world' (TO). This was expressed here in terms of the proportion of actual foreign investment to GDP as a proxy for a correlation between the rate of economic 'opening up' and city-level carbon emissions. It is clear that the use of foreign capital can have two effects on carbon emissions; on the one hand, some researchers have argued that foreign investment will result in the importation of more environmentally friendly technology to improve energy efficiency and therefore reduce carbon emissions, while others have argued that this variable improves the carbon emissions of energy utilization via the transformation of high energy consumption and industries within the host country.

\subsection{Data sources}

Because of a national top-down energy statistical system, Chinese consumption balance tables are only available at the regional level, with the exception of a handful of developed cities. Two approaches were therefore applied to address missing data in historical city-level 
carbon emissions, the use of existing energy statistics and the inversion of nighttime light data (Liu et al., 2012; Su et al., 2014; Wang and Liu, 2017). Thus, the IPCC greenhouse gas emissions inventory and energy consumption carbon emissions based on city-level energy consumption data were initially utilized, followed by DMSP/OLS nighttime light images, city night light data, and statistics that compare gross nighttime light values. These data were then fitted to corresponding carbon emission statistics. In order to ensure the reliability of our carbon emission simulation values, comparisons were carried out for cities at the prefectural-level cities based on available statistical data; results show that the relative error between simulated and calculated carbon emission values is $7.65 \%$. In other words, the accuracy of carbon emission proxies based on nighttime optical data is good and can be applied with relative accuracy to determine regional differences and impact mechanisms. In addition to city-level carbon emissions, other relevant data was extracted from the China City Statistical Yearbook, the China Statistical Yearbook for Regional Economy, and annual data compilations from relevant provinces and cities.

\section{Results and discussion}

\subsection{Regional differences and spatial spillover effects in city-level carbon emissions}

The initial focus of this study is on trends in city-level carbon emissions and the identification of regional differences. The data presented in Figure 1 reveal that city-level carbon emission per capita have increased year-by-year across China; between 1992 and 2013, national city-level carbon emissions per capita increased from $1.72 \mathrm{t}$ to $10.37 \mathrm{t}$, an average annual growth rate of $8.93 \%$. At the same time, however, values for the Gini coefficient reveal that regional differences in city-level carbon emissions have gradually narrowed over this time period; values for the Gini coefficient have gradually decreased from 0.72 in 1992 to 0.32 in 2013 . Thus, although national city-level per capita carbon emissions have increased, differences between cities have gradually narrowed.

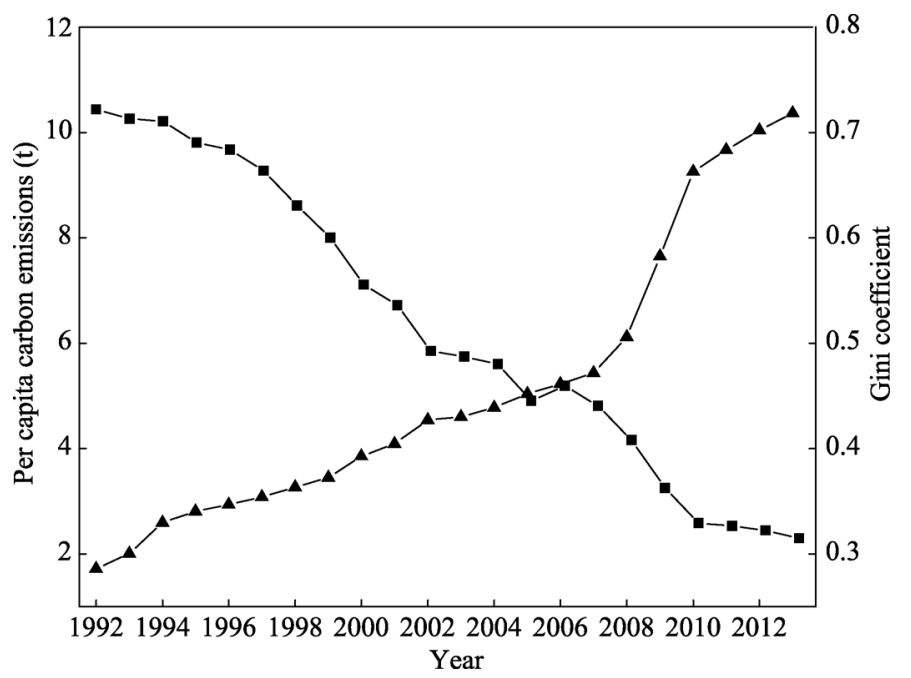

Figure 1 Average per capita carbon emissions at the city-level across China between 1992 and 2013 plotted alongside corresponding values for the Gini index 
The Tyler index is another method that is commonly applied to measure regional differences; indeed, this approach is preferable to the use of Gini coefficients because regional inequalities within, and between, groups can be identified and thus this index is often used to analyze these contributions relative to overall regional differences. Analysis of regional inequalities in city-level per capita carbon emissions across the eastern, central, western, and northeastern regions of China (Figure 2) reveals that between 1992 and 1995, per capita carbon emission differences in eastern cities made the largest contribution to overall differences in city-level per capita totals, followed by western, central, inter-regional areas, and the northeast. In contrast, between 1995 and 2013, the contribution rate of per capita carbon emissions from western Chinese cities was the largest and gradually increased, while this rate among eastern cities gradually reduced, and weak fluctuations were seen in the contributions from all other regions. Although the per capita carbon emissions of eastern cities gradually increased over this time period, differences between the values for different agglomerations gradually narrowed, indicating that both the energy consumption and industrial structures of cities in this region converged and gradually transitioned to renewable energy and service industry-dominated structures. At the same time, the per capita carbon emissions of cities in western China have increased, as have their divergences; these data reveal significant differences in the degree of fossil fuel-based energy dependence in western Chinese cities throughout their economic growth.

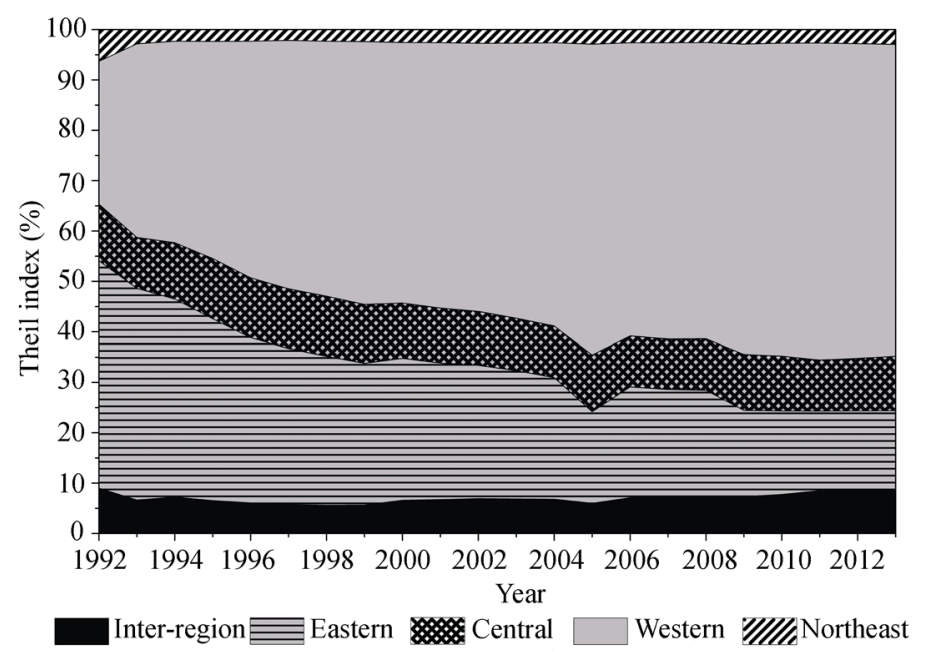

Figure 2 Contributions of regional inequalities within, and between, groups versus overall values of city-level per capita carbon emissions across China between 1992 and 2013

Although these results are clear, spatial correlations in data are not incorporated into the Gini coefficient, the Tyler index, or decomposition. This is important because the first law of geography notes that anything distributed in space is related to its associated attributes, and that this correlation is inversely proportional to spatial distance. Thus, if this spatial autocorrelation is neglected during analyses of the regional evolution of entities, our results will be seriously affected or biased. Spatial autocorrelations at different weights (i.e., $W_{1}, W_{2}$, and $W_{3}$ ) were therefore calculated, and results reveal global Moran's $I$ values that are all greater than zero and have significance not less than one percent given the $W_{1}$ and $W_{3}$ weight matrices; this indicates that city-level per capita carbon emissions are positively correlated 
spatially between high-value and low-value agglomeration areas. In contrast, however, values for global Moran's $I$ are not significant given the $W_{2}$ economic weight matrix; this result indicates that city-level per capita carbon emissions do not share spatial correlation characteristics with economic differences, but rather are autocorrelated with the geographical and synthetic geography-economy spaces. This global spatial autocorrelation reflects the overall relationships within these data, but often ignores atypical characteristics within local areas. A further aim of this article is therefore to explore the characteristics of spatial per capita carbon emission clustering within local cities via the monitoring of these autocorrelations. A scatter plot of national city-level per capita carbon emissions within a geospatial weight matrix for three representative years is shown in Figure 3; the vertical axis in this figure denotes the spatial lag of city-level per capita carbon emissions, while the horizontal axis denotes standardized city-level per capita emissions. These data show (Figure 3) that most Chinese cities are located within the first and third quadrants in this matrix, which further highlights the fact that per capita carbon emissions exhibit both significant high and low group characteristics.
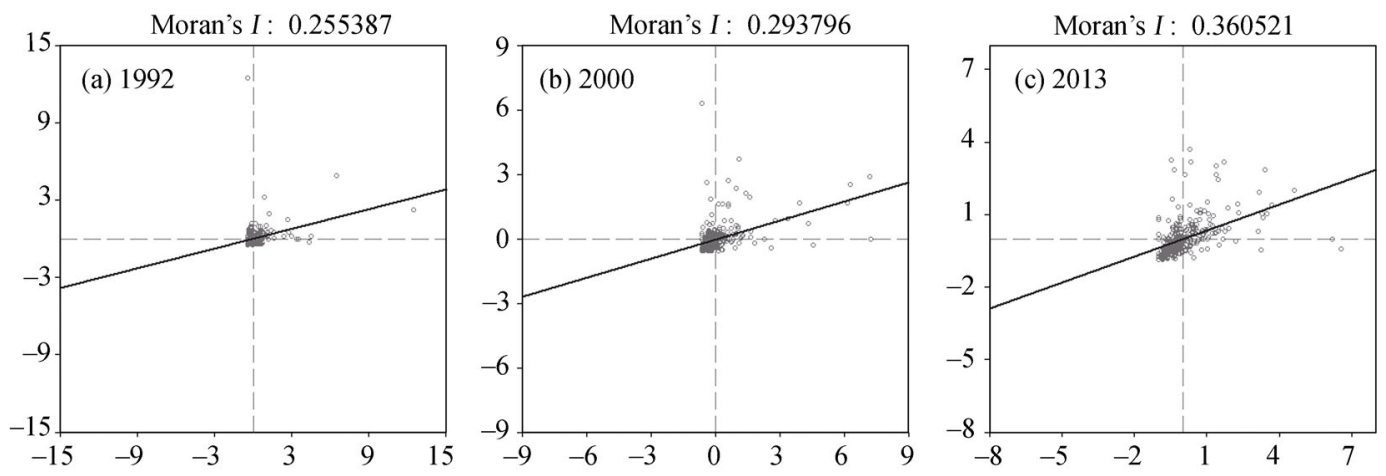

Figure 3 Scatter plots to show city-level per capita carbon emissions (Moran's I) within a geospatial weight matrix for three selected years

Although spatial autocorrelation analysis can reveal the global and local spatial clustering characteristics of city-level per capita carbon emissions, this approach does not incorporate spatial spillover effects. To address this, comparisons between the results of Markov-chain transitional matrices and their spatial counterparts will help to better understand the dynamic spatial spillover effects of city-level per capita carbon emissions. In the first place, city-level per capita carbon emissions were classified as either at a low level (i.e., per capita carbon emissions at the national average of $50 \%$ or below), at a medium-to-low level (i.e., per capita carbon emissions within the national average range between $51 \%$ and $100 \%$ ), at a medium-to-high level (i.e., per capita carbon emissions within the national average range between $101 \%$ and $150 \%$ ), or at a high level (i.e., per capita carbon emissions above the national average of $150 \%$ ). Next, in order to facilitate a comparative analysis, the research period encompassed by this study was divided into two, between 1992 and 2000 and between 2001 and 2013 (Figure 4 and Table 1). The elements on the diagonal in Table 1 denotes the probability that the type of city-level per capita carbon emissions remain unchanged, while all other components correspondingly indicate the probability of a change in the type of city-level per capita carbon emissions. All the diagonal elements in Table 1 are higher than 
their non-diagonal counterparts, which indicates that city-level carbon emissions are more likely to remain unchanged; indeed, the diagonal value is between 0.848 and 0.970 , indicating a minimum probability of $84.8 \%$ that city-level carbon emissions will not transfer. In contrast, the maximum value on this diagonal is 0.071 , indicating that the maximum probability of transformation in city-level carbon emissions is just $7.1 \%$; data further reveal that even if the city-level carbon emission type changes, this transition will occur between two adjacent types. Leaps forward in the development of city-level carbon emissions are barely present in these data as the probability of such a transfer is almost zero; a city with an initially high level of carbon emissions is also characterized by a $92.8 \%$ probability that this will be retained in subsequent years, and an associated probability of just $7.2 \%$ that this level will fall. In contrast, a city with an initially low level of carbon emissions also has a minimum $94.9 \%$ probability of maintaining this into the future alongside a maximum probability of just $5.1 \%$ that emissions will increase. These results also show that distinct types of city-level carbon emissions are also characterized by a spatial spillover effect that converges with the evolutionary process. In general, the probability of city-level carbon emissions increasing was $17.9 \%$ between 1992 and 2000, while the probability of a reduction was $19 \%$. Similarly, between 2001 and 2013, the probability of an increase was $13.5 \%$ and the probability of a reduction was $9.3 \%$. The presence of these two distinct phases in transfer results for city-level carbon emissions indicates that the negative spillover effect of the first phase is significantly higher than that of the second.

Table 1 Markov-chain transitional matrices for Chinese city-level per capita carbon emissions between 1992 and 2013

\begin{tabular}{|c|c|c|c|c|c|}
\hline & $n$ & $P(\leqslant 50 \%)$ & $\begin{array}{c}L \text { (between } 51 \% \\
\text { and } 100 \%)\end{array}$ & $\begin{array}{c}D \text { (between } 101 \% \\
\text { and } 150 \%)\end{array}$ & $R(\geqslant 150 \%)$ \\
\hline \multicolumn{6}{|c|}{ Between 1992 and 2013} \\
\hline$P$ & 3,098 & 0.957 & 0.042 & 0.000 & 0.000 \\
\hline$L$ & 2,002 & 0.023 & 0.936 & 0.040 & 0.000 \\
\hline$D$ & 872 & 0.006 & 0.054 & 0.873 & 0.068 \\
\hline$R$ & 1,252 & 0.005 & 0.006 & 0.034 & 0.955 \\
\hline \multicolumn{6}{|c|}{$1992-2000$} \\
\hline$P$ & 1,342 & 0.949 & 0.049 & 0.001 & 0.001 \\
\hline$L$ & 657 & 0.038 & 0.906 & 0.055 & 0.002 \\
\hline$D$ & 323 & 0.015 & 0.065 & 0.848 & 0.071 \\
\hline$R$ & 430 & 0.014 & 0.014 & 0.044 & 0.928 \\
\hline \multicolumn{6}{|c|}{ 2001-2013 } \\
\hline$P$ & 1,756 & 0.964 & 0.036 & 0.000 & 0.000 \\
\hline$L$ & 1,345 & 0.016 & 0.951 & 0.033 & 0.000 \\
\hline$D$ & 549 & 0.000 & 0.047 & 0.887 & 0.066 \\
\hline$R$ & 822 & 0.000 & 0.001 & 0.029 & 0.970 \\
\hline
\end{tabular}

Abbreviations: $P$, low level; $L$, medium-to-low level; $D$, medium-to-high level; $R$, high level.

The spatial Markov-chain transitional matrix calculated in this study for Chinese per-capita city-level carbon emissions is presented in Table 2. The data presented in Tables 1 and 2 reveal that the probability of an upward movement in per capita carbon emissions is higher in cities adjacent to other high level ones, while the probability of a downward transfer is low, and vice versa. Between 1992 and 2000, for example, the probability of an upward 
(a) $1992-2000$

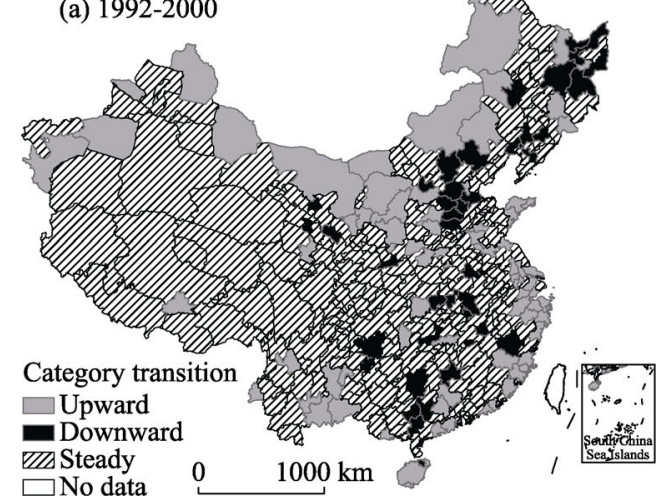

(b) 2001-2013

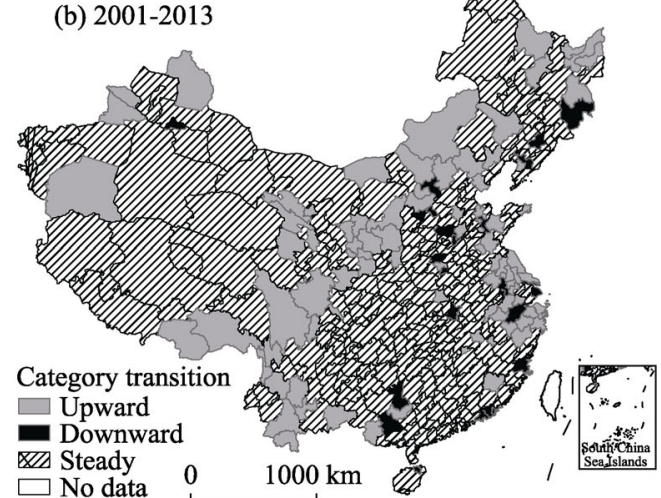

Figure 4 Spatial patterns in Chinese per capita carbon emissions at the city-level between 1992 and 2000 (a) and between 2001 and 2013 (b)

transfer in a low-level city was 0.051 , but this increased to 0.087 and 0.098 when an agglomeration was located adjacent to either a medium-to-low or medium-to-high level counterpart, and fell to 0.038 when two low level cities were located adjacent to one another. The probability of a high-level city downward transfer between 2001 and 2013 was 0.030 , but this fell to 0.007 when two cities of this type were located adjacent to one another. Thus, the spillover effect direction between city-level neighbors exerts a significant impact on the development of an agglomeration and leads to the phenomenon of developmental path dependence. In general, both standard and spatial Markov-chain models provide a realistic and clear theoretical basis to understand path dependence in city development.

Table 2 Spatial Markov-chain transitional matrices for Chinese city-level per capita carbon emissions between 1992 and 2013

\begin{tabular}{|c|c|c|c|c|c|c|c|c|c|}
\hline & & \multicolumn{4}{|c|}{ Between 1992 and 2000} & \multicolumn{4}{|c|}{ Between 2001 and 2013} \\
\hline & & $P$ & $L$ & $D$ & $R$ & $P$ & $L$ & $D$ & $R$ \\
\hline \multirow{4}{*}{$\mathrm{P}$} & $P$ & 0.962 & 0.038 & 0.000 & 0.000 & 0.972 & 0.028 & 0.000 & 0.000 \\
\hline & $L$ & 0.043 & 0.904 & 0.053 & 0.000 & 0.091 & 0.904 & 0.025 & 0.000 \\
\hline & $D$ & 0.057 & 0.096 & 0.836 & 0.011 & 0.000 & 0.112 & 0.864 & 0.024 \\
\hline & $R$ & 0.000 & 0.000 & 0.000 & 1.000 & 0.000 & 0.000 & 0.000 & 1.000 \\
\hline \multirow{4}{*}{$L$} & $P$ & 0.913 & 0.087 & 0.000 & 0.000 & 0.946 & 0.054 & 0.000 & 0.000 \\
\hline & $L$ & 0.027 & 0.926 & 0.047 & 0.000 & 0.042 & 0.942 & 0.016 & 0.000 \\
\hline & $D$ & 0.017 & 0.154 & 0.818 & 0.011 & 0.000 & 0.063 & 0.916 & 0.021 \\
\hline & $R$ & 0.000 & 0.000 & 0.066 & 0.934 & 0.000 & 0.000 & 0.064 & 0.936 \\
\hline \multirow{4}{*}{$D$} & $P$ & 0.902 & 0.098 & 0.000 & 0.000 & 0.868 & 0.132 & 0.000 & 0.000 \\
\hline & $L$ & 0.015 & 0.931 & 0.054 & 0.000 & 0.061 & 0.928 & 0.011 & 0.000 \\
\hline & $D$ & 0.000 & 0.139 & 0.813 & 0.038 & 0.000 & 0.088 & 0.884 & 0.018 \\
\hline & $R$ & 0.000 & 0.000 & 0.072 & 0.928 & 0.000 & 0.000 & 0.036 & 0.964 \\
\hline \multirow{4}{*}{$R$} & $P$ & 1.000 & 0.000 & 0.000 & 0.000 & 0.784 & 0.216 & 0.000 & 0.000 \\
\hline & $L$ & 0.015 & 0.931 & 0.054 & 0.000 & 0.075 & 0.914 & 0.011 & 0.000 \\
\hline & $D$ & 0.000 & 0.108 & 0.863 & 0.029 & 0.000 & 0.000 & 0.908 & 0.092 \\
\hline & $R$ & 0.000 & 0.000 & 0.013 & 0.987 & 0.000 & 0.000 & 0.007 & 0.993 \\
\hline
\end{tabular}

Abbreviations as in Table 1. 


\subsection{Factors influencing city-level carbon emissions and policies for their reduction}

The robust LM test was used in this study to compare spatial lag and the spatial error models and to determine which is more suitable for use in determining the key factors that influence city-level carbon emissions. Most previous studies of this type have compared the significance of the LM and robust LM statistics (LeSage et al., 2009); however, the data presented in Table 3 show that robust LM test results based on the spatial lag model are significantly higher than those derived from the spatial error model under the geospatial weight matrix $\left(W_{1}\right)$ and the geographically nested matrix of weight-economic weight $\left(W_{3}\right)$ which suggests that the former is more suitable for model parameter estimation than the latter. The results of the spatial lag model are therefore used as the basis for the analyses presented in this study, and it is also more effective to apply a system generalized distance estimation (Sys-GMM) approach to estimate a dynamic space panel model because of the endogenous problem of variables than to apply a general estimation method. The Sys-GMM approach also relies on time change trends in variables to automatically identify reasonable tools without having to specify a particular one.

Estimated results for the two spatial weight matrices, $W_{1}$ and $W_{3}$, are presented in Table 4. These data show that previous results published by Sargan and Arellano-Bond are in line with the requirements of Sys-GMM, and therefore corroborate the validity of the tool variables selected for use in this model. It is also noteworthy that when the sample tool variable is weak, this selection process might lead to an impact on, or cause a deviation from, Sys-GMM results. Results derived from a panel mixed least square method (PLOS) and panel fixed effect model (FE) are compared in this study to further corroborate Sys-GMM data; observations show that estimation coefficients derived from the PLOS method tend to transfer upwards, while those from the FE method tend to estimate downward result transfers. Thus, in cases where the estimated coefficients from Sys-GMM fall between the two, the validity of these results can be further corroborated. The data presented in Table 4 show that the city-level carbon emission time lag coefficient based on Sys-GMM is larger than that derived from PLOS estimations, and is smaller than that derived from FE; these results also show that Sys-GMM estimations are not anymore valid because of the choice of tool variables, and that coefficients based on this approach tend to be significantly higher than those from the other two methods. This suggests that the variable endogenous problem exerts an important effect on model estimation results. We therefore chose to apply a dynamic spatial panel data model to analyze the time reflection and spatial lag effects of city-level carbon emissions, while processing the variable endogenous problem via a Sys-GMM model. Further discussion is focused on the results estimated using the Sys-GMM model.

Table 3 LM test results using spatial panel models

\begin{tabular}{lccccc}
\hline \multirow{2}{*}{ LM test } & \multicolumn{2}{c}{$W_{1}$ weight matrix } & & \multicolumn{2}{c}{$W_{3}$ weight matrix } \\
\cline { 2 - 3 } \cline { 5 - 5 } & $\chi^{2}$ & $p$-value & $\chi^{2}$ & 0.000 \\
\hline No lag & 1675.474 & 0.000 & & 1684.328 & 0.002 \\
No lag (robust) & 25.856 & 0.000 & 26.732 & 1669.529 & 0.000 \\
No error & 1671.253 & 0.000 & & 14.967 & 0.169 \\
No error (robust) & 18.712 & 0.248 & & \\
\hline
\end{tabular}


The time effect results generated in this study show that the lag coefficient, $\theta$, for Chinese city-level per capita carbon emissions is both highly significant and positive with respect to geographic and geo-economic weights, which indicates the presence of a path dependence in these emissions; in other words, current levels of carbon emissions will have a certain impact on future ones. Results show that for every $1 \%$ increase in the current levels of carbon emissions, there will be a $0.7 \%$ increase in the next phase. Second, results show that the spatial lag coefficient, $\rho$, is also highly significant and positive with respect to geographic and geo-economic weights, which reflects the obvious spatial agglomeration characteristics of city-level per capita carbon emissions. Given $W_{1}$ and $W_{3}$ weight matrix settings, per capita carbon emissions will increase by about $1.08 \%$ for every $1 \%$ increase in neighboring cities. Third, the spatiotemporal lag coefficient, $\gamma$, of city-level per capita carbon emissions is significantly positive at the $1 \%$ level, again with respect to geographic and geo-economic weights; this indicates that a higher level of previous emissions in adjacent geographic or geo-economic areas will lead to a reduction in the emission levels of a given city.

This paper initially examined relationships in the EKC curve between economic growth and per capita carbon emissions. The data presented in Table 4 show that the primary coefficient of economic growth is significantly positive while the corresponding quadratic coefficient is significantly negative; these results are indicative of an inverted U-shaped relationship between city-level per capita carbon emissions and economic development. The per-capita carbon emission calculations presented in this study clearly reveal that most Chinese cities are still in a growth phase as their per capita GDP values are located to the left of the inflection point in this U-shaped curve; in other words, both carbon emission levels and the economic growth of these cities will continue to grow positively for some time into the future. The 'decoupling' phase between emissions and growth predicted by the traditional EKC hypothesis is not yet evident in these Chinese cities, but nevertheless further emphasizes the importance and urgency of both energy-saving and emissions reduction. The constraints of economic development have meant that growth is the primary focus for the vast majority of local governments; this is an issue because rapid economic growth is bound to lead to concomitant increases in carbon emissions. Cities cannot rely solely on the expense of economic growth to reduce their levels of carbon emissions, even in light of international and domestic pressure. This means that sustainable development should be emphasized in the future, and urban residents must be guided towards choosing low-carbon travel and green modes of consumption in order to reach a win-win situation of stable economic growth combined with a continued reduction in carbon emissions.

The negative effects of population density on city-level carbon emissions are also clear; in general, increases in population density influence carbon emissions in two main ways. The first of these is referred to as the agglomeration effect while the second is referred to as the scale effect. An increase in population density tends to lead to agglomeration effects, which increase the levels of technology, the shared proportion of public transport, and energy efficiency, while at the same time promoting the sharing of emission reduction facilities to reduce the release of carbon. At the same time, however, an increase in population density can also directly, or indirectly, lead to increasing energy consumption, the primary factor responsible for enhanced carbon emissions. A population density increase will also enhance traffic congestion, which also causes an increase in carbon emissions increase because of the 
inadequate combustion of motor vehicle fuel. The estimates presented in this study suggest that the agglomeration effect of population density is significantly higher than the scale effect; thus, any increase in energy efficiency brought about by population agglomeration is significantly greater than the corresponding increase in carbon emissions brought by a change in population scale. More attention should therefore be focused on enhancing the role of population agglomeration in energy efficiency in order to alleviate the impacts of associated scale effects on the future levels of carbon emissions.

Data reveal the significant negative effect of technological progress on city-level carbon emissions; progress in this area can have an impact via both production and emission reduction technologies. The former mainly acts on the productivity efficiency of various factors, while emission reduction technologies emphasize intensity; in other words, technological progress can promote reductions in energy intensity as well as lower carbon emissions, but also promote economic growth and increase carbon emissions. The results of this paper show that scientific expenditure across China has been effective in improving the levels of energy saving technology which has led to improvements in the efficiency of energy utilization, and has constrained city-level carbon emissions. This result also reminds us, however, to continue to increase investment in technological innovation and to improve research intensity as these variables play effective roles in both energy saving and the reduction of emissions.

An increase in the proportion of secondary industries across China has also had a significantly positive effect on city-level carbon emissions. As a result of the rapid development of industrialization, secondary industries, especially energy and high carbon intensive heavy industries, have developed rapidly to become the main driving force underlying Chinese economic development. Although positive, these industries are also the main contributors to city-level carbon emissions; these emission levels can be mitigated via industrial structural upgrades to phase out and eliminate out of date and traditional production techniques and replace them with high technological, value-added, intensive industries. The Chinese government promotes the optimization, transformation, and upgrade of industrial structures and has increased support for green industries as these are effective approaches to reduce carbon emissions.

Another way to significantly inhibit carbon emissions is to increase the density of the road network by more than $1 \%$; thus, given the same volume of traffic, the higher the ROAD, the lighter the traffic congestion, and complete combustion of motor vehicle fuel also becomes more likely. The development of convenient and efficient public transport networks should also be a future priority as this enhances transport capacity, and the government should vigorously promote and enhance the low-carbon travel awareness of urban residents. All of these initiatives are important measures that can reduce carbon emissions.

The results of this study show that the estimated coefficient of fixed asset investment across China is significantly positive at the $1 \%$ level. This indicates that an increase in this kind of investment has also raised carbon emission levels; indeed, the rapid growth of Chinese city-based economies is driven by investment and thus energy dependence on fossil fuels is also increasing. Fixed asset investment in China is also mainly concentrated on secondary industries, the most energy-dependent sector of the economy characterized by carbon 
emissions that are much larger than those of their primary or tertiary counterparts. Extensive investment in secondary industries is also unfortunately not conducive to industrial restructuring as injections of funds are mainly used to enhance the use of technology in production rather than green processes. This has therefore resulted in an expansion in production and has had a positive influence on city-level carbon emissions. Chinese city governments should screen FAIs, prioritize low-carbon options, and improve thresholds for environmental funding in order to more actively promote progress in green technologies. Policies such as these would also serve to enhance the role of technological progress in mitigating some of the scale effects associated with carbon emissions.

An overall increase in the openness of China has also had a significant effect on reducing city-level carbon emissions. Indeed, the use of foreign capital can improve environmental quality via income and technology spillover effects, while increases in non-indigenous investment tends to expand the scale of production and improve the income levels of local residents, enabling funds to guarantee energy savings and emission reductions. The ecological awareness and desire for high quality environments of residents will also be enhanced in concert with increasing income levels, and this informal environmental regulation will force improvements and consequent reductions in city-level carbon emissions. Foreign direct investment (FDI) also introduces new environmentally friendly products and technologies which drive associated spillover effects, enhance green application levels, and reduce Chinese carbon emissions. City governments should therefore incorporate low-carbon screening into their vetting of FDI in order to improve its environment threshold. Full advantage should be taken of both technological benefits and spillover effects in improving environmental quality, as an approach to actively develop the function of FDI in reducing carbon emissions.

Table 4 Estimated results from the dynamic spatial panel data models applied in this study

\begin{tabular}{|c|c|c|c|c|c|c|}
\hline \multirow{2}{*}{ Variable } & \multicolumn{3}{|c|}{$W_{1}$ weight matrix } & \multicolumn{3}{|c|}{$W_{3}$ weight matrix } \\
\hline & PLOS & $\mathrm{FE}$ & Sys-GMM & PLOS & $\mathrm{FE}$ & Sys-GMM \\
\hline $\ln \left(\mathrm{CE}_{\mathrm{t}-1}(\theta)\right)$ & $0.864^{* * *}$ & $0.266^{* * *}$ & $0.703^{* * *}$ & $0.864^{* * *}$ & $0.268^{* * *}$ & $0.702^{* * *}$ \\
\hline$\omega \ln (\mathrm{CE}(\rho))$ & $1.455^{* * *}$ & $1.622^{* * *}$ & $1.082^{* * *}$ & $1.396^{* * *}$ & $1.624^{* * *}$ & $0.179^{* * *}$ \\
\hline$\omega \ln \left(\mathrm{CE}_{\mathrm{t}-1}(\gamma)\right)$ & $0.688^{* * *}$ & $0.247^{* * *}$ & $0.726^{* * *}$ & $0.687^{* * *}$ & $0.249^{* * *}$ & $0.724^{* * *}$ \\
\hline $\ln (\mathrm{GDPPC})$ & $0.578^{*}$ & $0.642^{* * *}$ & $0.594^{* * *}$ & 0.580 & $0.638^{* * *}$ & $0.596^{* * *}$ \\
\hline $\ln (\mathrm{GDPPC})^{2}$ & $-0.017^{*}$ & $0.034^{* *}$ & $0.026^{* * *}$ & 0.019 & $0.035^{* *}$ & $0.025^{* * *}$ \\
\hline $\ln (\mathrm{POPD})$ & 0.142 & $-0.247^{* *}$ & $-0.386^{* * *}$ & 0.104 & $-0.246^{* *}$ & $-0.388^{* * *}$ \\
\hline $\ln (\mathrm{TC})$ & $-0.053^{* * *}$ & $-0.049^{* * *}$ & $-0.064^{* * *}$ & $-0.054^{* * *}$ & $-0.048^{* * *}$ & $-0.064^{* * *}$ \\
\hline $\ln (\mathrm{SIND})$ & $0.099^{* * *}$ & $0.163^{* * *}$ & $0.254^{* * *}$ & $0.104^{* * *}$ & $0.164^{* * *}$ & $0.255^{* * *}$ \\
\hline $\ln (\mathrm{ROAD})$ & $-0.067^{*}$ & $-0.123^{* * *}$ & $-0.154^{* * *}$ & $-0.066^{* *}$ & $-0.123^{* *}$ & $-0.155^{* * *}$ \\
\hline $\ln (\mathrm{FAI})$ & $0.057^{* * *}$ & $0.086^{* * *}$ & $0.066^{* * *}$ & $0.057^{* * *}$ & $0.085^{* * *}$ & $0.067^{* * *}$ \\
\hline $\ln (\mathrm{TO})$ & $-0.023^{*}$ & $-0.018^{* * *}$ & $-0.027^{* * *}$ & $0.023^{*}$ & $-0.019^{* * *}$ & $-0.028^{* * *}$ \\
\hline Sargan P & & & 0.675 & & & 0.663 \\
\hline $\mathrm{AR}(2) \mathrm{P}$ & & & 0.816 & & & 0.892 \\
\hline
\end{tabular}

Abbreviations: ${ }^{*}$, significant at the $10 \%$ level; ${ }^{* *}$, significant at the $5 \%$ level; ${ }^{* * *}$, significant at the $1 \%$ level. 


\section{Conclusions}

The analyses presented in this study are based on remote sensing data simulations of the carbon emissions of Chinese cities between 1992 and 2013. Regional differences in these emissions were initially analyzed using the Gini coefficient; results show that although per capita carbon emissions have increased over time, the differences between cities have gradually narrowed. Application of a decomposition model based on the Tyler index reveals that the differences among western Chinese cities have contributed to the largest extent to overall differences in carbon emissions, followed by contributions from eastern and central regions. The smallest contribution to overall emissions across China has been from interregional and northeastern regions. The application of three different spatial weight matrices shows that city-level carbon emissions across China are not characterized by the correlation features characteristic of economic development differences, but rather the presence of a group of high emission cities clustered within a geospatial and geo-economically integrated space. Dynamic analysis of classical Markov and spatial Markov matrices demonstrates that the evolution of city-level carbon emission types within China are also characterized by obvious features indicative of spatial spillover effects and path dependence, and that type transfers are significantly affected by the presence of adjacent cities. The probability of an upward transfer is enhanced when a city is located adjacent to one with higher-level emissions, while the reverse is true of downward transfers.

The application of a dynamic spatial panel data model in this study has enabled the quantitative identification of the key factors that influence city-level carbon emissions as well as a discussion of policies that could be applied to reduce emissions, given the constraints of time and spatial lag as well as the spatiotemporal hysteresis effect. The results of this study reveal the presence of a significant inverted U-shaped relationship between per capita carbon emissions and GDP, as well as the fact that levels of the former in most cities are still at a stage where they are certain to increase because of future economic growth. City-level per capita carbon emissions are also strongly influenced by secondary industry-oriented structures and an extensive increase in investment at this level. Although the results of this study show that the population agglomeration effect is higher than that of scale on city carbon emissions there is nevertheless a significant inhibitory effect even though its impact is small. Increases in the level of science and technology, openness, and road transportation network intensity all promote reduction in city-level carbon emissions. In future policy, it will be necessary to curb the factors that lead to increases while at the same time developing those that lead to decreases in order to effectively reduce city-level carbon emissions across China. The conclusions of this study are of great policy significance if we are to manage the low-carbon development of Chinese cities via socioeconomics. The national government should continue to optimize industrial structures, streamline extensive investment, increase the intensity research and development, and promote the accessibility of roads in order to realize future energy saving and emissions reduction targets.

\section{References}

Cheng Yeqing, Wang Zheye, Zhang Shouzhi et al., 2013. Spatial econometric analysis of carbon emission inten- 
sity and its driving factors from energy consumption in China. Acta Geographica Sinica, 68(10): 1418-1431. (in Chinese)

Deng Jixiang, Liu Xiao, Wang Zheng, 2014. Characteristics analysis and factor decomposition based on the regional difference changes in China's $\mathrm{CO}_{2}$ emission. Journal of Natural Resources, 29(2): 189-200. (in Chinese)

Fan Ying, Zhang Xiaobing, Zhu Lei, 2010. Estimating the macroeconomic cost of $\mathrm{CO}_{2}$ emission abatement in China based on multi-objective programming. Advances in Climate Change Research, 6(2): 130-135. (in Chinese)

Feng K S, Davis S J, Sun L X et al., 2015. Drivers of US $\mathrm{CO}_{2}$ emissions 1997-2013. Nature Communications, 6: 7714.

GEA, 2012. Global Energy Assessment: Toward a Sustainable Future. Cambridge, UK: Cambridge University Press.

He Xiaogang, Zhang Yaohui, 2012. Influence factors and environmental Kuznets curve relink effect of Chinese industry's carbon dioxide emission: Empirical research based on STIRPAT model with industrial dynamic panel data. China Industrial Economy, (1): 26-35. (in Chinese)

IEA, 2012. World Energy Outlook 2012. Paris: International Energy Agency (IEA).

IPCC, 2007. Summary for Policymakers of Climate Change: The Physical Science Basis. 2007-06-30. [2017-04-10]. https://www.ipcc.ch/pdf/assessment- report/ar4/wg1/ar4-wg1-spm.pdf.

LeSage J P, Pace R K, 2009. Introduction to Spatial Econometrics, Boca Raton: Taylor and Francis.

Liu Z, Feng K S, Davis S J et al., 2016. Understanding the energy consumption and greenhouse gas emissions and the implication for achieving climate change mitigation targets. Applied Energy, 184: 737-741.

Liu Z F, He C Y, Zhang Q et al., 2012. Extracting the dynamics of urban expansion in China using DMSP-OLS nighttime light data from 1992 to 2008. Landscape and Urban Planning, 106: 62-72.

$\mathrm{Pu}$ Yingxia, Ma Ronghua, Ge Ying et al., 2005. Spatial-temporal dynamics of Jiangsu regional convergence with spatial Markov-chain approach. Acta Geographica Sinica, 60(5): 817-826. (in Chinese)

Shao Shuai, Li Xin, Cao Jianhua et al., 2016. China's economic policy choices for governing smog pollution based on spatial spillover effects. Economic Research, (9): 73-88. (in Chinese)

$\mathrm{Su}$ Yongxian, Chen Xiuzhi, Ye Yuyao et al., 2013. The characteristics and mechanisms of carbon emissions from energy consumption in China using DMSP/OLS night light imageries. Acta Geographica Sinica, 68(11): 1513-1526. (in Chinese)

Su Y X, Chen X Z, Li Y et al., 2014. China's 19-year city-level carbon emissions of energy consumptions, driving forces and regionalized mitigation guidelines. Renewable and Sustainable Energy Reviews, 35: 231-243.

Wang Changjian, Wang Fei, Zhang Hongou, 2016a. The process of energy related carbon emissions and influencing mechanism research in Xinjiang. Acta Ecologica Sinica, 36(8): 2151-2163. (in Chinese)

Wang Changjian, Zhang Xiaolei, Zhang Hongou et al., 2016b. Influencing mechanism of energy-related carbon emissions in Xinjiang based on IO-SDA model. Acta Geographica Sinica, 271(7): 1105-1118. (in Chinese)

Wang Lei, 2014. Prediction of carbon emissions in Tianjin based on the input-output model. Ecological Economy, 30(1): 51-56. (in Chinese)

Wang S J, Fang C L, Guan X L et al., 2014a. Urbanization, energy consumption, and carbon dioxide emissions in China: A panel data analysis of China's provinces. Applied Energy, 136: 738-749.

Wang S J, Fang C L, Ma H T et al., 2014b. Spatial differences and multi-mechanism of carbon footprint based on GWR model in provincial China. Journal of Geographical Sciences, 24(4): 804-822.

Wang S J, Fang C L, Wang Y, 2016c. Spatiotemporal variations of energy-related $\mathrm{CO}_{2}$ emissions in China and its influencing factors: An empirical analysis based on provincial panel data. Renewable \& Sustainable Energy Reviews, 55: 505-515.

Wang Shaojian, Fang Chuanglin, Wang Yang et al., 2013. The directions and mechanisms of regional inequality in Guangdong province. Geographical Research, 32(12): 2244-2256. (in Chinese)

Wang S J, Fang C L, Wang Y et al., 2015a. Quantifying the relationship between urban development intensity and 
carbon dioxide emissions using a panel data analysis. Ecological Indicators, 49: 121-131.

Wang S J, Li Q Y, Fang C L et al., 2016d. The relationship between economic growth, energy consumption, and $\mathrm{CO}_{2}$ emissions: Empirical evidence from China. Science of the Total Environment, 542: 360-371.

Wang S J, Liu X P, 2017. China's city-level energy-related $\mathrm{CO}_{2}$ emissions: Spatiotemporal patterns and driving forces. Applied Energy, 200(15): 204-214.

Wang S J, Liu X P, Zhou C S et al., 2017. Examining the impacts of socioeconomic factors, urban form, and transportation networks on $\mathrm{CO}_{2}$ emissions in China's megacities. Applied Energy, 185: 189-200.

Wang Shaojian, Liu Yanyan, Fang Chuanglin, 2015b. Review of energy-related $\mathrm{CO}_{2}$ emission in response to climate change. Progress in Geography, 34(2): 151-164. (in Chinese)

Wang Shaojian, Wang Yang, Zhao Yabo, 2014c.GIS-based multi-scale and multi-mechanism research on regional inequality in Guangdong province. Scientia Geographica Sinica, 30(10): 1184-1192. (in Chinese)

Wang Shaojian, Wang Yang, Zhao Yabo, 2015c. Spatial spillover effects and multi-mechanism for regional development in Guangdong province since the 1990s. Acta Geographica Sinica, 70(6): 965-979. (in Chinese)

Wang Y, Li G D, 2017. Mapping urban $\mathrm{CO}_{2}$ emissions using DMSP/OLS 'city lights' satellite data in China. Environmental and Planning A, 49(2): 248-251.

Yao C R, Feng K S, Hubacek K, 2014. Driving forces of $\mathrm{CO}_{2}$ emissions in the G20 countries: An index decomposition analysis from 1971 to 2010. Ecological Informatics, 26: 93-100.

Yuan Lu, Pan Jiahua, 2013. Disaggregation of carbon emission drivers in Kaya identity and its limitations with regard to policy implications. Advances in Climate Change Research, 9(3): 210-215. (in Chinese)

Zhang Y, 2009. Structural decomposition analysis of sources of decarbonizing economic development in China: 1992-2006. Ecological Economics, 68(8/9): 2399-2405.

Zhang Zhenghua, Peng Diyun, 2013. Review on the empirical research on the impact factors of China's carbon dioxide emissions. Ecological Economy, (6): 50-54. (in Chinese)

Zheng Changde, Liu Shuai, 2011. Empirical research of carbon emission and economic growth in China based on the spatial econometric analysis. China Population, Resources and Environment, 21(5): 80-86. (in Chinese)

Zhu Yuen, Li Lifen, He Sisi et al., 2016. Peak year prediction of Shanxi Province's carbon emissions based on IPAT modeling and scenario analysis. Resources Science, 38(12): 2316-2325. (in Chinese) 